\title{
25-Gauge Vitrectomy Combined with Half-Fluence Photodynamic Therapy for the Treatment of Juxtapapillary Retinal Capillary Hemangioma: A Case Report
}

\author{
Cesare Mariotti $^{\mathrm{a}} \quad$ Alfonso Giovannini $^{\mathrm{a}} \quad$ Michele Reibaldi $^{\mathrm{b}}$ \\ Michele Nicolai $^{a} \quad$ Andrea Saitta $^{a}$ \\ ${ }^{a}$ Ophthalmology Department, Polytechnic University of Marche, Ancona, and \\ ${ }^{b}$ Department of Ophthalmology, University of Catania, Catania, Italy
}

\section{Key Words}

25-Gauge vitrectomy · Juxtapapillary retinal capillary hemangioma - Photodynamic treatment · von Hippel-Lindau disease

\begin{abstract}
A rare case of juxtapapillary retinal capillary hemangioma $(J R C H)$ with tractional macular detachment that was managed successfully with minimally invasive 25-gauge vitreoretinal surgery, followed by two sessions of half-fluence photodynamic treatment (PDT) with good visual outcome, is presented. A 13-year-old female patient had progressive deterioration of the vision of her right eye due to the presence of tractional macular detachment associated with JRCH in von Hippel-Lindau (VHL) disease. A 25-gauge sutureless vitreoretinal surgery was successfully performed. Two months following surgery, the JRCH was treated with two sessions of half-fluence PDT at an interval of 4 months. Visual acuity improved from a preoperative level of 20/50 to 20/25 24 months postoperatively. Resolution of the tractional macular detachment, reduction of papillomacular area fluid, and reduction in size of the JRCH were observed during the follow-up period. No serious adverse events were observed. Small-gauge vitreoretinal surgery followed by PDT can be effective and safe in relieving tractional macular detachment and recovering useful visual acuity in JRCH in VHL disease.
\end{abstract}


Mariotti et al.: 25-Gauge Vitrectomy Combined with Half-Fluence Photodynamic

Therapy for the Treatment of Juxtapapillary Retinal Capillary Hemangioma

\section{Introduction}

Retinal capillary hemangioma (RCH) is the most frequent manifestation of von HippelLindau (VHL) disease [1]. RCHs are congenital hereditary capillary angiomatous hamartomas of the retina and optic nerve head. RCHs can have different locations: at or near the optic disc (juxtapapillary RCHs, JRCHs; up to $15 \%$ of cases), and at the midperiphery and/or posterior pole (peripheral RCHs) $[1,2]$. In general, JRCHs are treated if they are progressive or if these lesions affect visual acuity. JRCHs are difficult to treat, particularly if they are located temporally. Several treatments have been proposed, but no single modality in the treatment of JRCHs has been particularly effective [3, 4].

Vitreoretinal surgery is indicated when JRCHs are complicated with epiretinal membrane (ERM) formation and serous and tractional retinal detachment of the macula, which leads to a poor visual prognosis. Only a few cases of vitreoretinal surgery have been reported to date [5-7]. In 2011, Gaudric et al. [7] stated that 20-gauge vitreoretinal surgery had improved the vision in half of their 23 patients with RCHs and they suggested that surgery may be preceded or followed by conventional laser coagulation or photodynamic therapy (PDT). Effective treatment using PDT for RCHs in the posterior pole has been documented in several reports $[8,9]$.

We report a case of progressive JRCH in VHL disease associated with a tractional macular detachment that underwent successful combined therapy with 25-gauge vitreoretinal surgery and PDT. To the best of our knowledge, this is the first reported treatment for paramacular RCHs with small-gauge vitreoretinal surgery before PDT.

\section{Case}

A 13-year-old girl presented with reduced right-eye vision and metamorphopsia for 2 months. She had a negative ocular and medical history. Since the patient was adopted, we were unable to obtain information about her family history. At presentation, her best corrected visual acuity (BCVA) was 20/32 and 20/20 for the right and left eyes, respectively. At fundus examination of the right eye, a round circumscribed orange elevated lesion was revealed at the optic disc, covered by thick epiretinal membrane that extended to the macula, with juxtapapillary subretinal fluid and macular edema surrounding the lesion (fig. 1a). Fundus autofluorescence (FAF) in the right eye demonstrated a round, slightly hypoautofluorescent lesion at the optic disc, surrounded by an indistinct hyperautofluorescent elevated area masquerading the retinal vessels, with some markedly hyperautofluorescent dots (fig. 1b). Spectral domain optical coherence tomography (SD-OCT) in the right eye confirmed a roundish elevated mass at the level of the optic disc, which was covered and surrounded by thick epiretinal membrane, and the presence of serous and tractional macular detachment (fig. 1c). Left-eye fundus examination revealed two smaller reddish lesions: one was juxtapapillary and the second was in the peripheral inferior retina. SD-OCT in the left eye showed a flattened retina. Angiography revealed the capillary nature of the lesions and shunting of blood from the arterial to the venous side of the circulation in the region of the peripheral tumor.

A diagnosis of bilateral JRCH was made. A systemic evaluation of the patient, including magnetic resonance imaging of the brain, computed tomography of the abdomen, and measurement of urinary vanillylmandelic acid levels, revealed cysts affecting the kidneys. Genetic studies revealed a mutation in the VHL tumor-suppressor gene VHL, confirming the diagnosis of VHL disease. 
After a 6-month period of observation, her BCVA decreased to 20/50 and metamorphopsia in the right eye continued to increase in association with an expansion of the lesion at fundus examination. A minimally invasive 25-gauge three-port pars plana vitrectomy using valved trocars was performed in the right eye in order to remove the posterior hyaloid membrane and macular traction, and to reduce and displace the hemangioma from the optic disc. Following core vitrectomy, triamcinolone acetonide suspension was injected above the posterior pole to provide clear visualization of the posterior hyaloid membrane. The fibrotic glial capsule of the hemangioma was removed completely in order to carefully disconnect the tumor from the optic disc and to displace it partially from the head of the optic disc. Considering the exophytic nature of the lesion, an excision of the tumor was impossible. High-energy laser photocoagulation and air tamponade were performed in order to stop the bleeding from the capillary hemangioma. At postoperative fundus examination, the JRCH appeared smaller and dislocated from a great part of the optic disc head.

Two months after surgery, half-fluence PDT was performed. The rationale for the use of half-fluence PDT $\left(25 \mathrm{~J} / \mathrm{cm}^{2}\right)$ was to minimize the risk of ischemic optic neuropathy [4]. Six months postoperatively, a second treatment with half-fluence PDT was performed for the presence of persistent subretinal fluid in the macula on SD-OCT.

Up to the last follow-up at 24 months, the BCVA of the patient's right eye had improved to $20 / 25$ and was stable. The JRCH appeared smaller in size with a regression of subretinal fluid (fig. 2a). FAF imaging revealed a hypoautofluorescent round area dislocated from the optic disc and surrounded by a hypoautofluorescent area (fig. 2b). Serous macular detachment disappeared with a replacement of a normal macular morphology, as documented by SD-OCT (fig. 2c). There have been no serious adverse events recorded to date, at the final 24 months of follow-up.

\section{Discussion}

$\mathrm{RCH}$ is a peculiar vascular tumor at the optic disc and posterior pole. Although the clinical course of JRCH is variable, it tends to cause progressive deterioration of vision. Cases of spontaneous relapse have been described, even though this is uncommon [1]. RCH can be very laborious to treat, in particular when the lesions are large and multiple [2,3]. In addition, juxtapapillary lesions are often recalcitrant to treatment. There are several treatment options, such as laser photocoagulation, cryotherapy, brachytherapy, diathermy, transpupillary thermotherapy, PDT, and surgical excision $[2,4,6,8-10]$. However, the effectiveness of these treatments in inducing regression of a lesion is still under debate.

Although there are no established guidelines, vitreoretinal surgery is therefore still an option to consider for the treatment of progressive cases of RCH [5-7, 11, 12]. The first reported case of RCH excision was in 1983, by Peyman et al. [10], who resected the wall of the tumor. More recently, vitreoretinal surgery was performed to remove an ERM as a complement to previous laser therapy, cryotherapy, or PDT [5, 7, 11, 12]. Last, fibrovascular proliferation from JRCH was also treated surgically in some cases [4, 6]. In 2002, Majji [11] reported a case of paramacular RCH treated and managed successfully with laser photocoagulation, followed by pars plana vitrectomy and removal of epiretinal tractional membranes.

In 2011, Gaudric et al. [7] stated that 20-gauge vitreoretinal surgery had improved the vision in half of their 23 patients and they suggested that surgery may be preceded or followed by conventional laser coagulation or PDT. 
PDT has been described to be an effective treatment of vascular tumors, with regression of $\mathrm{RCH}$, resolution of macular edema, and improvement of visual acuity, although its application for juxtapapillary lesions is limited by vaso-occlusive effects $[8,13]$. It is known that laser photocoagulation can be correlated with poor visual outcomes, because of irreversible damage to the nerve fiber layer, with consequent altitudinal defects and central scotoma in the visual field. Indeed, PDT guarantees preservation of adjacent tissue and the retinal nerve fiber layer, although it can result in shrinkage of the epiretinal membrane and worsening of visual acuity due to vitreoretinal traction [13]. Furthermore, for larger tumors, as noted above, verteporfin might only be active at the surface of the tumor, without closing the deeper tumor vessels, and hence the importance of PDT as a second procedure. Recently, Fong et al. [9] proposed combined therapy with intravitreal ranibizumab injection and PDT 1 week before pars plana vitrectomy and ERM peeling for the treatment of JRCHs.

In the last years, the minimally invasive sutureless transconjunctival pars plana vitrectomy (23-gauge and 25-gauge) has progressed so much that it has become the approach of choice for most vitreoretinal procedures. The advantages over 20-gauge procedures come primarily from the reduction of postoperative inflammation at the sclerotomy site, less manipulation of periocular tissues and less surgical time, leading to speedier recovery and a decreased rate of surgical complications $[14,15]$.

In our case, the JRCH was located in the papillomacular area, and vitreoretinal surgery was necessary because of progressive tractional detachment of the macula. Minimally invasive 25-gauge vitreoretinal surgery was effective and safe in removing epiretinal tractional membranes. The main goal of the surgery as first treatment was to remove macular traction and the thick fibrotic capsula of the JRCH. The second goal of the surgery was to reduce the angioma in size and to displace it partially from the head of the optic disc in order to minimize the risk of ischemic optic neuropathy after PDT, improving the efficacy of treatment.

After the second PDT, SD-OCT showed resolution of the macular exudation and reduction of papillomacular area fluid. We also observed a reduction in size and regression of the JRCH, which was stable at the last follow-up of 24 months.

In summary, combination therapy with small-gauge vitreoretinal surgery and PDT can be effective in the treatment of progressive JRCHs in VHL disease. However, longer follow-up periods and greater numbers of cases are needed to confirm the effectiveness of this combination therapy.

\section{Disclosure Statement}

The authors have no conflicts of interest to declare.

\section{References}

1 McCabe CM, Flynn HW Jr, Shield CL, et al: Juxtapapillary capillary hemangiomas: clinical features and visual acuity outcome. Ophthalmology 2000;107:2240-2248.

-2 Magee MA, Kroll AJ, Lou PL, Ryan EA: Retinal capillary hemangiomas and von Hippel-Lindau disease. Semin Ophthalmol 2006;21:143-150.

-3 Singh AD, Nouri M, Shields CL, Shields JA, Perez N: Treatment of retinal capillary hemangioma. Ophthalmology 2002;109:1799-1806.

4 Garcia-Arumi J, Sararols LH, Cacero L, et al: Therapeutic options for capillary papillary hemangiomas. Ophthalmology 2000;107:48-54.

5 McDonald HR, Schatz H, Johnson RN, et al: Vitrectomy in eyes with peripheral retinal angioma associated with traction macular detachment. Ophthalmology 1996;103:329-335. 


\begin{tabular}{l|l}
\hline \multicolumn{2}{l}{ Case Rep Ophthalmol 2014;5:162-167 } \\
\hline DOI: $10.1159 / 000363564$ & $\begin{array}{l}\text { C 2014 S. Karger AG, Basel } \\
\text { www.karger.com/cop }\end{array}$ \\
\hline
\end{tabular}

Mariotti et al.: 25-Gauge Vitrectomy Combined with Half-Fluence Photodynamic

Therapy for the Treatment of Juxtapapillary Retinal Capillary Hemangioma

6 Kreusel KM, Bechrakis NE, Neumann HP, Foerster MH: Pars plana vitrectomy for juxtapapillary capillary retinal angioma. Am J Ophthalmol 2006;141:587-589.

7 Gaudric A, Krivosic V, Duguin G, Massin P, Giraud S, Richard S: Vitreoretinal surgery for severe retinal capillary hemangiomas in Von Hippel-Lindau disease. Ophthalmology 2011;118:142-149.

8 Papastefanou VP, Pilli S, Stinghe A, Lotery AJ, Cohen VM: Photodynamic therapy for retinal capillary hemangioma. Eye (Lond) 2013;27:438-442.

-9 Fong AH, Li KK, Wong D: Intravitreal ranibizumab, photodynamic therapy, and vitreous surgery for the treatment of juxtapapillary retinal capillary hemangioma. Graefes Arch Clin Exp Ophthalmol 2011;249:625627.

$\rightarrow 10$ Peyman GA, Rednam KR, Mottow-Lippa L, Flood T: Treatment of large von Hippel tumors by eye-wall resection. Opthalmology 1983;90:840-847.

-11 Majji AB: Paramacular Von Hippel angioma with tractional macular detachment. Ophthalmic Surg Lasers 2002;33:145-147.

12 Schwartz PL, Fastenberg DM, Shakin JL: Management of macular puckers associated with retinal angiomas. Ophthalmic Surg 1990;21:550-556.

-13 Schmidt-Erfurth UM, Kusserow C, Barbazetto IA, Laqua H: Benefits and complications of photodynamic therapy of papillary capillary hemangiomas. Ophthalmology 2002;109:1256-1266.

14 Thompson JT: Advantages and limitations of small gauge vitrectomy. Surv Ophthalmol 2011;56:162-172.

-15 Reibaldi M, Rizzo S, Avitabile T, Longo A, Toro MD, Viti F, Saitta A, Giovannini A, Mariotti C: Iatrogenic retinal breaks in 25-gauge vitrectomy under air compared with the standard 25-gauge system for macular diseases. Retina 2014, Epub ahead of print.
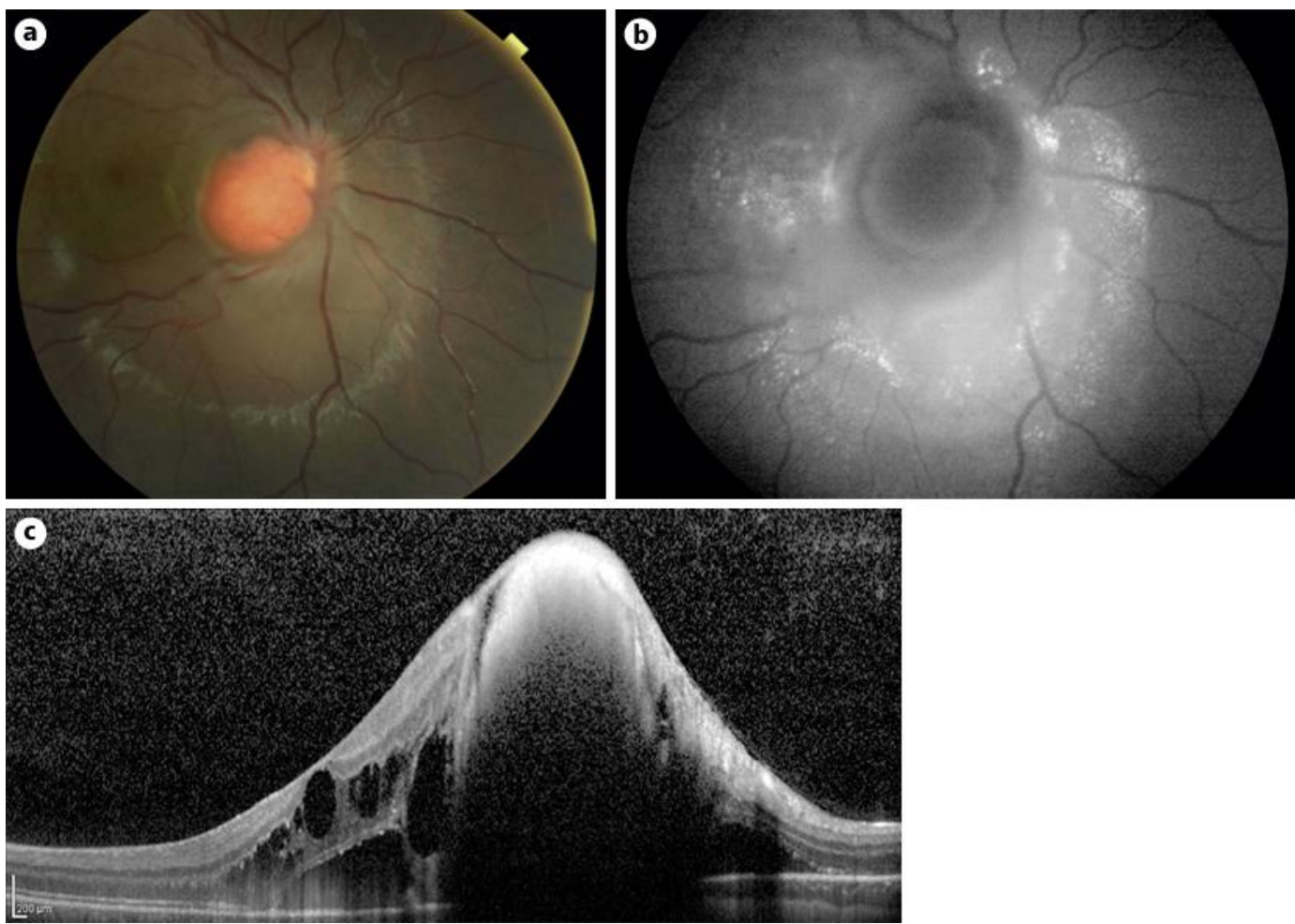

Fig. 1. a At right-eye fundus examination, a 13-year-old girl showed a round circumscribed orange elevated lesion completely covering the optic disc and associated with juxtapapillary subretinal fluid. b At FAF, there was a round, slightly hypoautofluorescent lesion at the optic disc, surrounded by an indistinct hyperautofluorescent elevated area with the appearance of retinal vessels, with some markedly hyperautofluorescent dots detected at FAF. c SD-OCT showed the presence of a thick epiretinal membrane and serous and tractional retinal detachment of the macula. The patient's BCVA was 20/50. 
Case Reports in

Ophthalmology

\begin{tabular}{l|l}
\hline Case Rep Ophthalmol 2014;5:162-167 \\
\hline DOI: 10.1159/000363564 & $\begin{array}{l}\text { ○ 2014 S. Karger AG, Basel } \\
\text { www.karger.com/cop }\end{array}$ \\
\hline
\end{tabular}

Mariotti et al.: 25-Gauge Vitrectomy Combined with Half-Fluence Photodynamic Therapy for the Treatment of Juxtapapillary Retinal Capillary Hemangioma
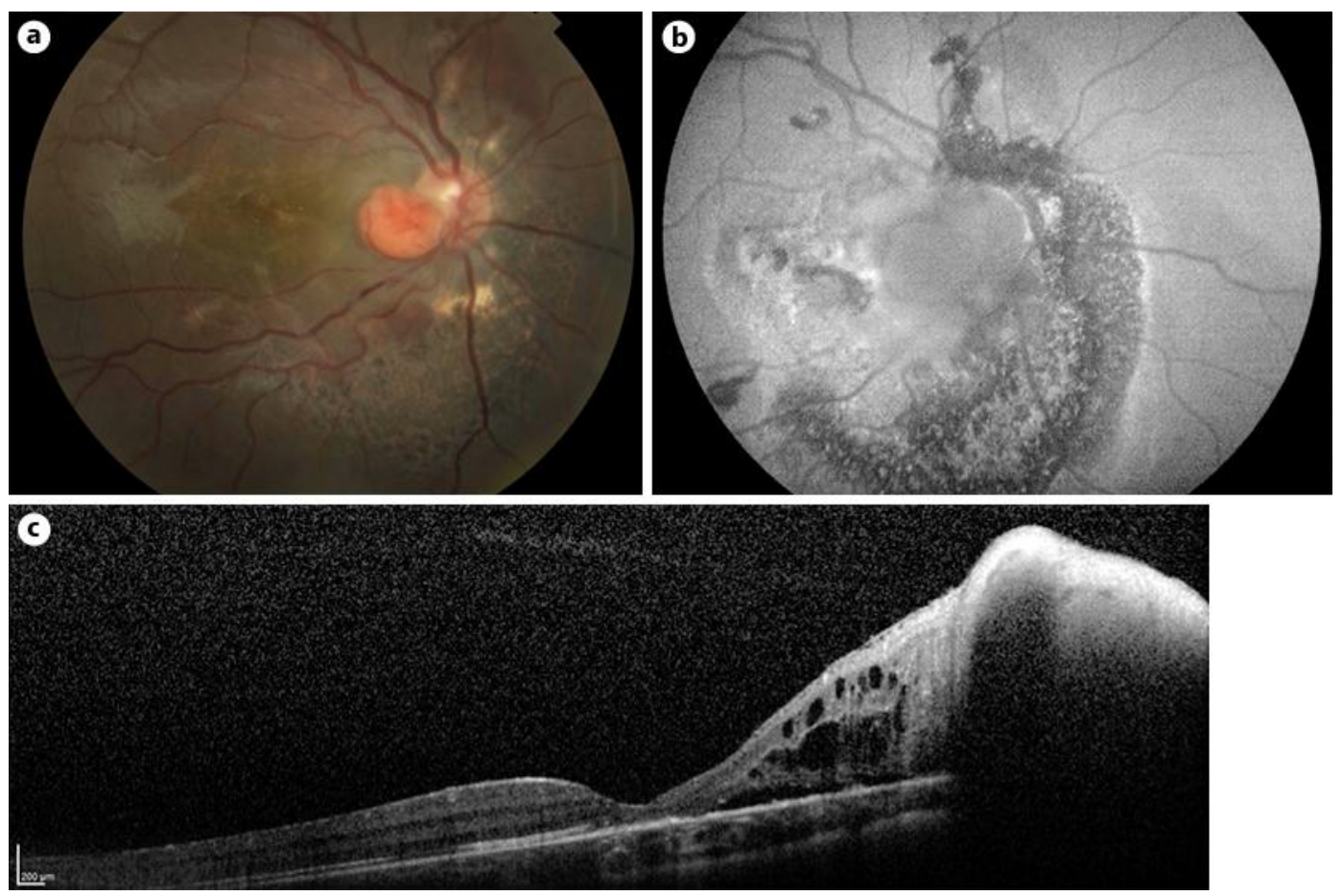

Fig. 2. a Postoperative fundus examination showed a reduction in size of the JRCH, and the optic nerve head appeared almost completely free from the lesion. The JRCH was stable in size for the whole duration of follow-up. b Twenty-four months postoperatively, FAF imaging revealed a hypoautofluorescent round area dislocated from the optic disc and surrounded by a hypoautofluorescent area. c At the last follow-up, SD-OCT showed resolution of the macular exudation and reduction of papillomacular area fluid. The patient's BCVA had improved to 20/25. 